\title{
A point in many triangles
}

\author{
Boris Bukh
}

Submitted: Mar 3, 2006; Accepted: May 19, 2006; Published: May 29, 2006

Mathematics Subject Classification: 52C10, 52C30

\begin{abstract}
We give a simpler proof of the result of Boros and Füredi that for any finite set of points in the plane in general position there is a point lying in $2 / 9$ of all the triangles determined by these points.
\end{abstract}

\section{Introduction}

Every set $P$ of $n$ points in $\mathbb{R}^{d}$ in general position determines $\left(\begin{array}{c}n \\ d+1\end{array}\right) d$-simplices. Let $p$ be another point in $\mathbb{R}^{d}$. Let $C(P, p)$ be the number of the simplices containing $p$. Boros and Füredi [2] constructed a set $P$ of $n$ points in $\mathbb{R}^{2}$ for which $C(P, p) \leq \frac{2}{9}\left(\begin{array}{l}n \\ 3\end{array}\right)+O\left(n^{2}\right)$ for every point $p$. They also proved that there is always a point $p$ for which $C(P, p) \geq \frac{2}{9}\left(\begin{array}{l}n \\ 3\end{array}\right)+O\left(n^{2}\right)$. Here we present a new simpler proof of the existence of such a point $p$.

\section{Proof}

Let $P$ be a set of $n$ points in the plane. By the extension of a theorem of Buck and Buck [3] due to Ceder [4] there are three concurrent lines that divide the plane into 6 parts each containing at least $n / 6-1$ points in its interior. Denote by $p$ the point of intersection of the three lines. Every choice of six points, one from each of the six parts, determines a hexagon containing the point $p$.

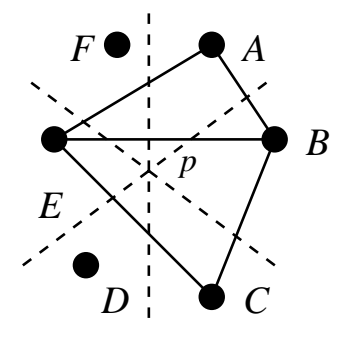

Figure 1: a) $p \in A B E$ or $p \in B C E$
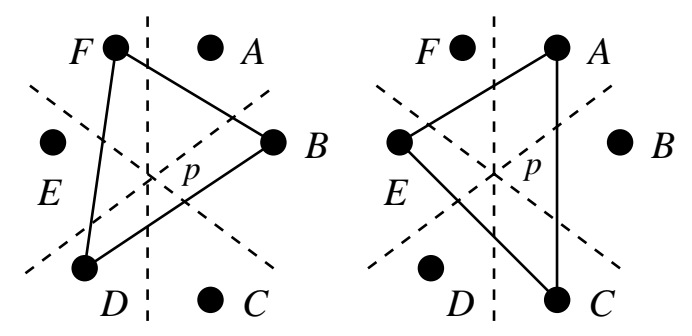

b) $p \in A C E$ and $p \in B D F$

Among the $\left(\begin{array}{l}6 \\ 3\end{array}\right)=20$ triangles determined by the vertices of the hexagon, at least 8 triangles contain the point $p$. Indeed, from each of the six pairs of triangles situated as in 
Figure 1a we get one triangle containing $p$. In addition, $p$ is contained in both triangles of the Figure 1b. Therefore, by double counting, the number of triangles containing $p$ is at least

$$
\frac{8(n / 6-1)^{6}}{(n / 6-1)^{3}}=\frac{2}{9}\left(\begin{array}{l}
n \\
3
\end{array}\right)+O\left(n^{2}\right)
$$

For the sake of completeness we include a sketch of a proof of the modification of the theorem of Buck and Buck that we used above.

Proposition 1. Let $\mu$ be a finite measure absolutely continuous with respect to the Lebesgue measure on $\mathbb{R}^{2}$. Then there are three concurrent lines that partition the plane into six parts of equal measure.

The partition theorem for the finite set of point $P$ follows by letting $\mu$ be the restriction of the Lebesgue measure to the union of tiny disks of equal size centered at the points of $P$. Since $P$ is in general position, none of the three lines passes through more than two of the disks.

Proof sketch. The given measure can be made into one which gives every open set a strictly positive measure, and which differs little from the given one. Proving the result for the latter, and using a compactness argument, one is through. Hence we can assume the property mentioned, and we normalize the total measure of the plane to 1 .

Let now $\mathrm{u}$ be a unit vector. There is a unique directed line $L(u)$ pointing in the direction $\mathrm{u}$ and cutting the plane in two parts of measure $1 / 2$. For any point $P$ on $L(u)$ there are six unique rays from $P$, denoted $A(u, P), \ldots, F(u, P)$ in clockwise order, splitting the plane in sectors of measure $1 / 6$, with $A(u, P)$ in the direction $u$. Note that $L(u)$ is the union of $A(u, P)$ and $D(u, P)$. When $P$ moves along $L(u)$ in the direction $u$, the ray $B(u, P)$ will turn counterclockwise in a continuous way, becoming orthogonal to $L(u)$ at some point. As the clockwise turning $E(u, P)$ behaves in the same way, there will be a unique $P^{*}(u)$ such that $B\left(u, P^{*}(u)\right)$ and $E\left(u, P^{*}(u)\right)$ form a line.

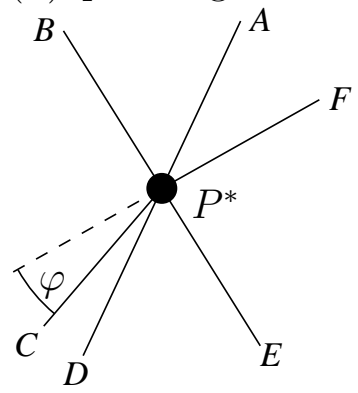

Figure 2: Six rays

The line $L$, the point $P^{*}$ and the six rays from $P^{*}$ clearly depend continuously on $u$. In particular the angle $\varphi(u)$ one must turn $C\left(u, P^{*}(u)\right)$ counterclockwise to complete $F\left(u, P^{*}\right)$ to a line varies continuously. But for any $u$, we have $C\left(-u, P^{*}(-u)\right)=$ $F\left(u, P^{*}(u)\right)$, and hence $\varphi(-u)=-\varphi(u)$. This shows that for some $v$ the angle $\varphi(v)$ vanishes and the rays $C\left(v, P^{*}(v)\right)$ and $F\left(v, P^{*}(v)\right)$ form a line. This finishes the proof.

For no dimension higher than 2 the optimal bounds for $C(P, p)$ are known. Bárány [1] showed that there is always a point $p$ for which $c(P, p) \geq(d+1)^{-d}\left(\begin{array}{c}n \\ d+1\end{array}\right)+O\left(n^{d}\right)$.

Acknowledgement. I thank the referee for comments that resulted in much improved proof of proposition 1 . 


\section{References}

[1] I. Bárány, A generalization of Carathéodory's theorem, Discrete Math. 40 (1982), 141-152.

[2] E. Boros and Z. Füredi, The number of triangles covering the center of an $n$-set, Geom. Dedicata 17 (1984), 69-77.

[3] R. C. Buck and E. F. Buck, Equipartition of convex sets, Math. Mag. 22 (1949), 195-198.

[4] J. G. Ceder, Generalized sixpartite problems, Bol. Soc. Mat. Mexicana (2) 9 (1964), 28-32. 